\title{
The Classification of Breast Calcification using Texture Feature of Fractal-based and Gabor Wavelet-based
}

\author{
Po-Tsun Liu, Chiun-Li Chin, Jing-Shian Chan, Hao-Hung Tsai
}

\begin{abstract}
According to the opinions of specialized doctors, being able to accurately classify breast calcification is very important, and with this information available, only then medical treatments can be applied properly. However, for any delay treatment or misdiagnosis, it is very likely as the key attributed to the fatal death of the patients. Currently, there are a lot of researches on the development of many methods with application of mammographic for classification of breast calcification out there already. However, in this paper, mammography image is used to classify breast calcification. And, we use a cubic curve contrast enhancement method to enhance image contrast. Next, we use Gabor wavelet and Fractal-based to extract texture feature on the breast image. Finally, we further to input these features into Back-propagation neural network method for classification. Next, we will make classification of these different features, as well as to put a link among them, in order to get better accuracy for classification. Experimental result shows our method has a good accuracy, and be able to precisely help the doctors for recognizing the breast calcification whether they are good or not. In addition, it can be verified that the accuracy rate of our method is up to $89.55 \%$.
\end{abstract}

Keywords Mammographic $\cdot$ breast calcification · cubic curve contrast enhancement Fractal-based · Gabor Wavelet

\author{
Hao-Hung Tsai $(\bowtie)$ \\ Department of Medical informatics, Chung Shan Medical University, Taichung, Taiwan \\ E-mail: jojonnngg@gmail.com \\ Po-Tsun Liu \\ Department of Medical informatics, Chung Shan Medical University, Taichung, Taiwan \\ Chiun-Li Chin \\ Department of Medical informatics, Chung Shan Medical University, Taichung, Taiwan \\ Jing-Shian Chang \\ Department of Medical informatics, Chung Shan Medical University, Taichung, Taiwan
}




\section{Introduction}

Breast cancer is ranked first among the leading causes of cancer affecting females [1]. In the paper, from the experiences of specialized doctors, we know that recognizing benign or malignant status among all breast calcifications is very important. There are two system are developed to assist doctor. One is called computer aided diagnosis and the other is called computer aided detection [2]. Concerning image segmentation and specification of regions of interest (ROIs), several methods have been proposed such as classical image filtering and local thresholding [3], techniques based on mathematical morphology [4], wavelet analysis [5] and multiscale analysis based on a specialized Gaussian and Peitgen [6]. From the survey of related work, we understand that there are many researchers to work on the enhancement and segmentation of breast image. But for recognizing breast calcification whether they are benign or malignant, it is rarely to be find. Hence, according to the radiologist suggestion, we tried to solve this problem using image processing and pattern recognition technology. There are five chapters in this paper. The earlier part of the first section is Introduction. In order to description is the following sections, in section 2 is a detailed description of our proposed complete procedure. In section 3 is experimental results. In section 4 is conclusions.

\section{Materials and Methods}

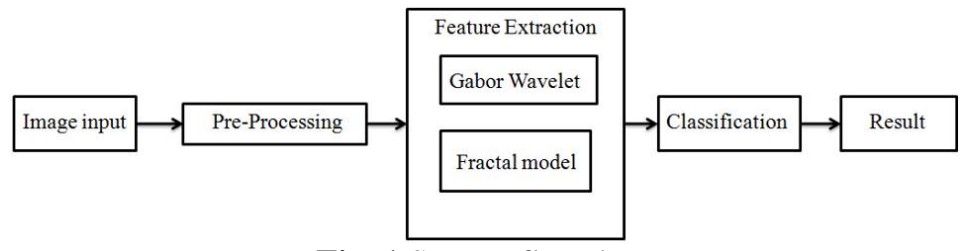

Fig. 1 System flowchart

In the paper, we use mammography images with DICOM format for analysis and processing, all of which are 8 bites images at pixel size of $2560 \times 3328$. The system process flow chart offered by us is shown in Fig. 1 . The system is divided into three main parts. Firstly, the system will work on preprocessing of the images and cutting the region of calcification. Afterwards, the system will perform feature extraction and analysis by Fractal. The goal is to obtain some useful features which can recognize breast calcification whether they are benign or malignant. Finally, we will 
enter these features extracted and analysis into Back propagation neural network (BPNN) method for classification [10].

\subsection{Preprocessing}

The preprocessing can be divided into three steps. First, we use Gaussian smoothing filter to remove noise. Next, the image projection method is used to obtain the breast part in the original image. Fig. 3 (a) shows the original image. There is a red lines in Fig. 3 (a). This red line are obtained by image brightness distinction method that we proposed. Fig. 3 (b) shows the result of image segmentation. Finally, in the past, for most of the doctors, if they want to view any medical images, they will use an approach called "window method [11] to increase on the degree of contrast for images, for example, viewing a region with brain stroke, they will then put a set of parameters; window center is set as $40 \mathrm{HU}$ and the window width (W) is set as 80 , however, for this approach, it is lack of flexibility, instead in the chapter, we will take adoption of cubic curve method [12] for contrast adjustment on images due the approach is with nice responsive effects in its sensibility to light changes, and its formula is shown as (1). The adaptive cubic curve is shown in Fig. 2. After preprocessing, we can enhance the region of interested (ROIs) from the original image in Fig. 3 (c) and (d).

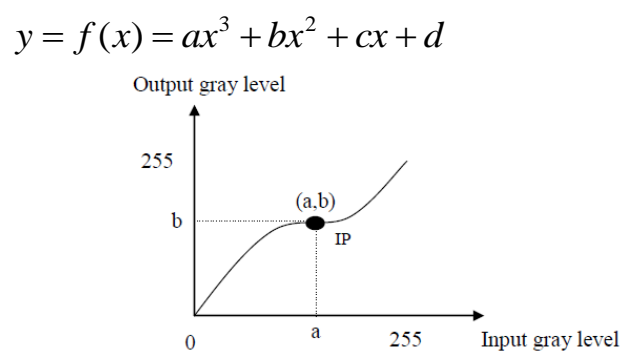

Fig. 2 Adaptive cubic curve for enhancing image contrast
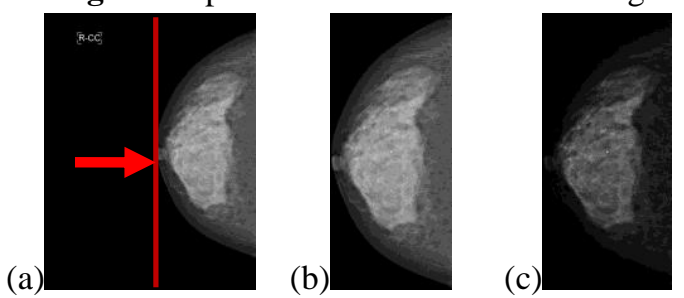

(d)

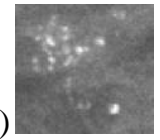

Fig. 3 (a) is original breast image, (b) is preprocessed image by image projection method, and (c) is the result image obtained by cubic curve contrast enhancement method, (d) the image of ROI 


\subsection{Feature Extraction}

In this paper, Gabor Wavelet and Fractal Dimension are used to extract the texture feature of image. First, Gabor wavelet transform is an extension of the Fourier transform, widely used in formal dimension. dimension value and the gray value normalization allows images to reduce the impact of changes in light, The difficulty of reducing the processing. And it's defined on:

$$
g(\vec{x})=\frac{k^{2}}{\sigma^{2}} \cdot e^{\frac{k^{2} \cdot\|\vec{x}\|^{2}}{2 \sigma^{2}}} \cdot\left[e^{i k \vec{x}}-e^{\frac{\sigma^{2}}{2}}\right]
$$

To obtain even with odd two different parts from the basic Gabor wavelet, and use the mean value and standard deviation for feature selection, we got five different features, respectively, MEM, SEM, MOM, SOM and TM.

$$
\mathrm{MEM}=\frac{\sum_{\mathrm{e}=1}^{\mathrm{x}} \mathrm{x}_{\mathrm{e}}}{\mathrm{x}}
$$

Where $\mathrm{e}$ is number of mean-even values. And $\mathrm{x}$ is the number of the matrix value.

$$
\mathrm{SEM}=\sqrt{\frac{\sum\left(x_{e}-x\right)^{2}}{n-1}}
$$

Where $\mathrm{n}$ is number of sample value.

$$
\mathrm{MOM}=\frac{\sum_{0=1}^{\mathrm{x}} \mathrm{x}_{0}}{\mathrm{x}}
$$

Where $o$ is number of mean-odd values.

$$
\begin{gathered}
\mathrm{SOM}=\sqrt{\frac{\sum\left(x_{0}-x\right)^{2}}{n-1}} \\
\mathrm{TM}=\frac{M E M+S E M+M O M+S O M}{4}
\end{gathered}
$$

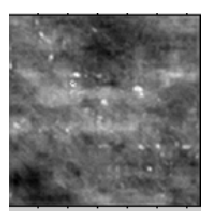

(a)

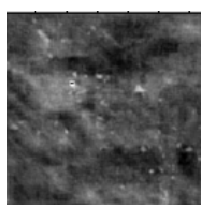

(b)

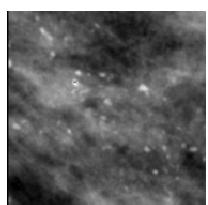

(c)

Fig. 4 (a) image of even, (b) image of odd, (c) original, (d) reconstructed

Then we reconstructed two image, compared with even image and odd image, show Fig. 4 (a), (b) and (c), (d) show the image of original and 
reconstructed. Then we know even the change is similar but it still made our feature more batter than original image. The secend method is based on fractal dimension. We hypothesize the fractal dimension correlates with image roughness. Fractal-based texture analysis was introduced by Pentland in 1984[16]. Fractal characteristics can be divided into two parts Self-similarity and scale of different sexual. The former refers to the fractal geometry of the system has invariant under changes observe a designated area. Zoomed a ROI the overall shape of the object the same thing the latter refers to no specific scale fractal geometry in terms of fractal collection whether it is larger or smaller seemingly irregular pattern actually has a certain symmetry.

$$
\left\{\mathrm{f}_{\mathrm{i}}: \mathrm{x} \rightarrow \mathrm{x} \mid \mathrm{i}=1,2, \ldots, \mathrm{n}\right\}, \mathrm{n} \in \mathrm{N}
$$

In definition, the number of iterations for a group of Han limited space contraction and contraction mapping for the $\mathrm{x}$ at equation 8 .

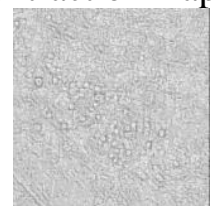

(a)

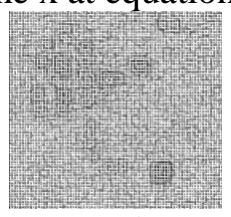

(b)

Fig. 5 (a) Show a calcification image after fractal analysis, (b) show different patients of calcification image after Gabor wavelet with fractal analysis

After fractal analysis, the texture will be better and obvious in Fig. 5, than we can know if the image got a good texture and the classification result will be upgrade.

\subsection{Classification}

In this study we choose Back-propagation neural network (BPNN) for classification and it's a kind of supervised learning networks, used in the diagnosis, prediction, classification, in this study, the prediction also uses back-propagation neural network to construct it. The different of the traditional perceptron network is BPNN made the following improvements: (a) Hidden layer (b) Instead smooth differentiable transfer function.

Back propagation neural network architecture diagram is optimized using the steepest descent method, solving the iteration of the optimum value, and joining hidden layer processing neurons, makes the optimization problem can be adjusted by way of argument, which gives a 
more accurate solution. Advantages are as follows: Learning precision, recall fast, and output values may be continuous discrete values, and can handle highly complex nonlinear function synthesis problems, applicability is quite extensive. We have give input and learning until only the best result.

\section{Experimental Results}

We use the mammography images of 12 patients to perform experiment. And there are 24 images of benign and 32 images of malignant in the paper. In order to evaluate the accuracy of our proposed system, firstly we will request professional doctor of radiology to help us to select training samples. These training samples can be classified two classes. One is benign breast calcifications. The other is malignant breast calcifications. Fig. 6 (a) to (c) show some benign breast calcification images. Fig. 6 (d) to (f) show some malignant breast calcification images. We can figure out that calcifications always easy to be find but hard to be resolution.

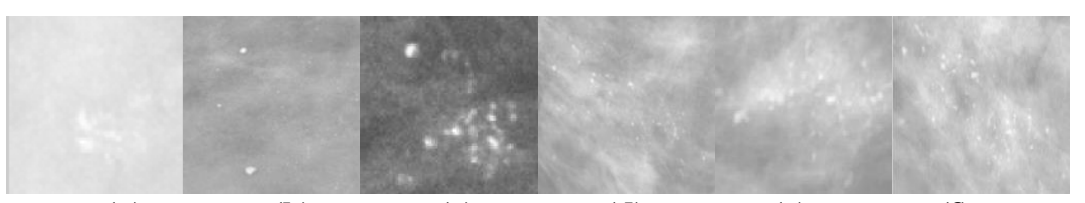

(a)

(b)

(c)

(d)

(e)

(f)

Fig. 6 (a) to (c) are benign breast calcification images, and (d) to (f) are malignant breast images
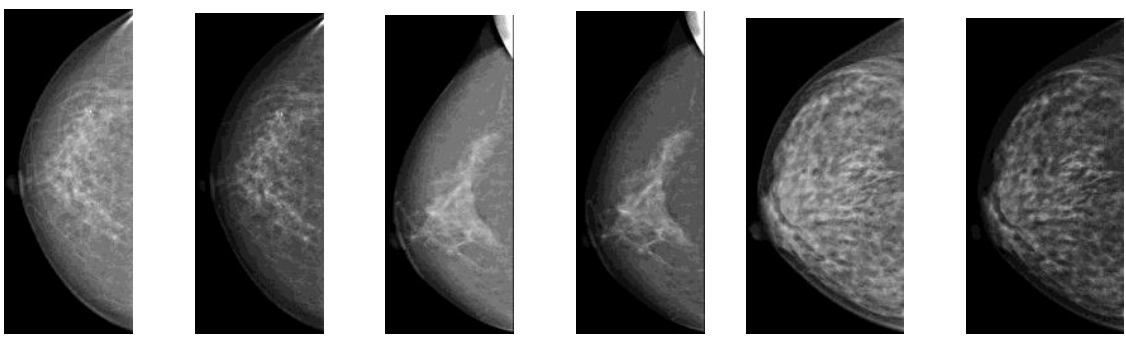

Fig. 7 The result of cubic curve contrast enhancement

Fig. 7 shows the result of cubic curve contrast enhancement. For calculating the performance of our proposed method, we use two evaluated method. First, the statistic evaluated indices are used, including detection rate $(D R)$, false alarm rate $(F A R)$, and correction rate $(C R)$. They are calculated with indications as TPN, FPN, TNN and FNN, respectively. Their equations are defined as the follows. $D R=(T P N) /$ number of 
malignant cases $; F A R=(F P N) /$ number of benign cases $; C R=(T P N+$ $T N N) /$ total number of cases. All three values can be calculated, and their results are as shown in Table 1 . We let 10 doctor tested our proposed system. The goal is to calculate average successful rate. This result shows our proposed system have $90 \%$ accuracy. Compare the result of Gabor wavelet and Gabor wavelet with fractal-analysis, In Table 2 show that accuracy was very greatly. It proved our proposed were useful.

Table 1 Classification Result

\begin{tabular}{|c|c|c|c|c|c|c|}
\hline TPN & FPN & TNN & FNN & DR\% & FAR\% & CR\% \\
\hline 18.25 & 3.12 & 31 & 4.23 & 88.2 & 11.5 & 89.55 \\
\hline
\end{tabular}

Table 2 Compared Classification Result

\begin{tabular}{|c|c|c|}
\hline CLASSIFEATURE & GABOR WAVELET & $\begin{array}{c}\text { GABOR WAVELET WITH } \\
\text { FRACTAL-ANALYSIS }\end{array}$ \\
\hline ACCURACY & $72 \%$ & $89.55 \%$ \\
\hline
\end{tabular}

\section{Conclusions}

In the recent years, there are more and more academic researches on the examinations of enhancement or extraction of breast calcification. But for researching the recognition breast calcification whether they are benign or malignant, it is rare. Hence, we use two feature extraction Gabor Wavelet and Fractal-based with BPNN classifier to recognize the breast calcificaiton image. From experimental result, we understand that our proposed system have $89.55 \%$ accuracy. It means that we proposed this system has good ability and better than the single Gabor wavelet. It could help doctor to judge the class of breast calcification image.

\section{References}

1. R. Nishikawa, (2007). Current status and future directions of computer-aided diagnosis in mammography. In Computerized Medical Imaging and Graphics, vol 31, pp 224-235. 
2. R.M. Rangayyan, F.J. Ayres \& J.E.L. Desautels, (2007). A review of computer-aided diagnosis of breast cancer: Toward the detection of subtle signs. In Journal of the Franklin Institute, vol. 344, no. 3, pp. 312-348.

3. R.C. Gonzalez, R.E, (2002). Woods, second edition, Prentice-Hall, Englewood Cliffs. Digital Image Processing, NJ.

4. B. Senthil kumar, G. Umamaheswari, (2011). A Novel Edge Detection Algorithm for the Detection of Breast Cancer. European Journal of Scientific Research, vol. 53, pp. 51-55.

5. Zikuan Chen, Ruola Ning, (2004). Breast volume denoising and noise characterization by 3D wavelet transform. Computerized Medical Imaging and Graphics, vol. 28, pp. 235-246.

6. Mohamed Meselhy Eltoukhy, Ibrahima Faye, Brahim Belhaouari Samir, (2010). Breast cancer diagnosis in digital mammogram using multiscale curvelet transform. Computerized Medical Imaging and Graphics, vol. 34, pp. 269-276.

7. Essam A. Rashed, Ismail A. Ismail , Sherif I. Zaki, (2007). Multiresolution mammogram analysis in multilevel decomposition. Pattern Recognition Letters, vol. 28, pp. 286-292.

8. Zhiyun Xue, Sameer Antani and L. Rodney Long et al., (2012). Window Classification of Brain CT Images in Biomedical Articles. AMIA Annu Symp Proc, vol. 2012, pp. 1023-1029.

9. Chin-Teng Lin and Chiun-Li Chin, (2006). Using Fuzzy Inference and Cubic Curve to Detect and Compensate Backlight Image. International Journal of Fuzzy Systems, vol. 8, pp. 2-13.

10.Przelaskowski A., Sklinda K. and Bargieł P. et al., (2008). Improved early stroke detection:Wavelet-based perception enhancement of computerized tomography exams. Computers in Biology and Medicine, vol. 37, pp. 524-533.

11.Mark Polak, Hong Zhang and Minghong Pi, (2009). An evaluation metric for image segmentation of multiple objects. Image and Vision Computing, vol. 27, pp. 1223-1227.

12.Amar Khoukhi and Syed Faraz Ahmed, (2011). A genetically modified fuzzy linear discriminant analysis for face recognition. Journal of the franklin institute, vol. 348, pp. 2701-2717.

13.João Batista Florindo, Odemir Martinez BrunoCorresponding, (2012). Fractal descriptors based on Fourier spectrum applied to texture analysis. Journal of Physica A: Statistical Mechanics and its Applications, vol. 391, pp. 4909-4922. 